\title{
Complicações clínicas na gravidez: uma revisão sistemática de estudos com gestantes brasileiras
}

\author{
Clinical complications during pregnancy: \\ a systematic review of studies with pregnant Brazilian women
}

Dixis Figueroa Pedraza (http://orcid.org/0000-0002-5394-828X) ${ }^{1}$

Anahi Cézar de Lima Lins (https://orcid.org/0000-0003-2983-0051) ${ }^{1}$

${ }^{1}$ Universidade Estadual da Paraíba. Av. das Baraúnas 351, Campus Universitário, Bodocongó. 58109-753

Campina Grande PB Brasil. dixisfigueroa@gmail.com

\begin{abstract}
The scope of this paper was to describe the main clinical complications among pregnant Brazilian women reported in the literature, identifying the most relevant exposure factors and their outcomes in maternal and child health. This is a systematic review of articles published between 2010 and 2018. Three reviews and 36 empirical articles were included; mental disorders ( $n=9)$, infectious diseases $(n=9)$ and severe maternal morbidity $(n=8)$ were the main clinical complications during pregnancy. In addition, four articles focused on determining the main conditions or causes of hospitalization, with a higher frequency of Urinary Tract Infection, anemia and hypertensive diseases. Maternal age at reproductive extremes, lower schooling, socioeconomic vulnerability, racial characteristics and the use of public health services were the most relevant exposure factors. Unfavorable health outcomes associated with clinical complications in pregnancy were verified in the eight articles with this profile. The frequency of pregnant women with potential life-threatening clinical complications is high in the country, highlighting the importance of primary care coverage and the implementation of interventions to reduce and prevent adverse maternal and child outcomes.
\end{abstract}

Key words Complications during pregnancy, Morbidity, Maternal mortality
Resumo Objetivou-se descrever as principais complicações clínicas em gestantes brasileiras relatadas na literatura, identificando os fatores de exposição mais relevantes e seus desfechos na saúde materno-infantil. Foi realizada uma revisão sistemática de artigos publicados entre 2010 e 2018. Foram incluídos três revisões e 36 artigos empiricos; transtornos mentais $(n=9)$, doenças infecciosas ( $n=9)$ e morbidade materna grave $(n=8)$ foram as principais complicações clínicas na gravidez. Ainda, quatro artigos enfocaram a determinação das principais afecções ou causas de internação, citando-se com maiores frequências a Infecção do Trato Urinário, a anemia e as doenças hipertensivas. Idade materna nos extremos reprodutivos, menor escolaridade, vulnerabilidade socioeconômica, características raciais e uso de serviços públicos de saúde foram os fatores de exposição mais relevantes. Resultados de saúde desfavoráveis associados a complicações clínicas na gravidez foram verificados nos oito artigos com esse perfil. A frequência de gestantes com complicações clínicas potenciais de ameaça à vida é elevada no país, evidenciando a importância da cobertura da atenção básica e a implantação de intervenções para sua redução e prevenção de desfechos maternos e infantis adversos.

Palavra-chave Complicações na gravidez, Morbidade, Mortalidade materna 


\section{Introdução}

A morbimortalidade materna, neonatal e fetal, constituem importantes indicadores da saúde materna e infantil, representando eventos de grande magnitude no Brasil. Estudos têm mostrado altas frequências de morbidade gestacional, como doenças hipertensivas, infecções e hemorragias, além de piores desfechos da gestação ${ }^{1,2}$. Ainda, relata-se o aumento dos casos de Near Miss Materno (NMM), os quais podem contribuir na morbimortalidade perinatal ${ }^{3-5}$. Essas condições refletem a falta de acesso aos serviços de saúde e as condições socioeconômicas desfavoráveis $^{1,2}$.

Nessa conjuntura, a assistência pré-natal destaca-se por permitir a identificação e o manejo de condições clínicas e comportamentais de risco nas gestações ${ }^{6}$. Assim, cuidados pré-natais de qualidade desempenham um papel importante na proteção e na redução de eventos adversos à saúde no período gestacional ${ }^{7}$.

No ano 2000, o Ministério da Saúde criou o Programa de Humanização no Pré-Natal e Nascimento (PHPN), que preconizou os parâmetros mínimos para uma atenção pré-natal adequada, com o objetivo de reduzir as elevadas taxas de morbimortalidade materna e perinatal do Brasil, ampliar o acesso, qualificar as consultas e promover o vínculo entre assistência ambulatorial e parto ${ }^{6}$. Posteriormente, a Rede Cegonha foi instituída como estratégia com o objetivo de estruturar e organizar a atenção à saúde materno-infantil, assegurando à mulher o direito ao planejamento reprodutivo e à atenção humanizada à gravidez, parto e puerpério, como garantindo acesso, acolhimento e resolutividade ${ }^{8}$. Tanto o PHPN quanto a Rede Cegonha destacam a importância de exames clínicos e laboratoriais como forma de garantir os cuidados necessários durante a fase gestacional e a qualidade da atenção ${ }^{6,8}$. Nessa perspectiva, esse trabalho tem como objetivo descrever as principais complicações clínicas em gestantes brasileiras relatadas na literatura, identificando os fatores de exposição mais relevantes e seus desfechos na saúde materno-infantil.

\section{Métodos}

Trata-se de uma revisão sistemática de literatura sobre complicações clínicas na gravidez em gestantes brasileiras. Para a realização desta revisão utilizou-se como protocolo as orientações PRISMA ${ }^{9}$. Dois revisores trabalharam de forma independente, na condução do trabalho. As discrepâncias entre os mesmos foram resolvidas por consenso.

A questão norteadora proposta para o estudo foi a seguinte: Quais as principais complicações clínicas em gestantes brasileiras relatadas na literatura, seus fatores de exposição mais relevantes e desfechos na saúde materno-infantil?

\section{Critérios de elegibilidade}

Consideraram-se elegíveis estudos contendo informações sobre complicações clínicas na gravidez, publicados entre 2010 e 2018. Foram considerados os registros nos idiomas inglês, espanhol e português; e documentos científicos publicados na forma de artigos originais cujos resultados procederam de estudos com gestantes brasileiras.

\section{Fontes de informações}

Os estudos foram identificados nas bases de dados da Biblioteca Virtual em Saúde (BVS), Scientific Electronic Library Online (SciELO) e $\mathrm{Na}$ tional Library of Medicine (PubMed). A busca foi realizada em 4 de fevereiro de 2019.

\section{Estratégia de busca}

Foram considerados, inicialmente, todos os documentos contendo o descritor em Ciências da Saúde "complicações na gravidez" ("pregnancy complications"). No PubMed, a busca adicionou o descritor "Brasil" ("Brazil"). Posteriormente, os documentos foram filtrados eliminando-se registros em idiomas diferentes do inglês, espanhol ou português; monografias, dissertações e teses; e artigos teóricos e de opinião. Para o cômputo do total de estudos identificados, verificou-se eventuais duplicações desses estudos entre as bases de dados, sendo cada artigo contabilizado somente uma vez.

\section{Seleção dos estudos}

Os estudos identificados foram submetidos a processo de triagem e, mediante leitura dos títulos e resumos, foram eliminados se (i) realizados em outros países que não o Brasil, (ii) não foram realizados com gestantes, e (iii) não fizeram menção a complicações clínicas na gravidez. Após o processo de triagem, os registros elegidos foram submetidos aos critérios de inclusão e exclusão, procedendo-se a leitura e a análise criteriosa do texto completo. 
Para inclusão nesta revisão, consideraram-se os artigos empíricos que abordassem complicações clínicas na gravidez no Brasil e os artigos de revisão sistemática sobre o tema incluindo nas suas análises estudos com população brasileira. Foram excluídos os artigos enquadrados nos seguintes critérios: revisões não sistemáticas, qualitativos, relatos de caso, descritivos, que não utilizaram amostras representativas, com foco em gestantes com alguma condição patológica específica e sem análises de associação entre exposições e complicações clínicas na gravidez ou entre estas e desfechos materno-infantis.

As listas de referências bibliográficas dos artigos incluídos foram analisadas com o objetivo de identificar outros possíveis estudos de interesse (busca reversa). Os artigos selecionados a partir de consulta às referências bibliográficas foram submetidos aos mesmos critérios de elegibilidade, previamente descritos.

\section{Extração dos dados}

As informações selecionadas para caracterizar os artigos que consideraram as complicações clínicas como desfecho foram: autor, ano de publicação, avaliação da qualidade, objetivos, delineamento, local de estudo, tamanho da amostra, exposições de estudo, complicações clínicas de interesse e respectivas ocorrências, e principais resultados (exposições associadas às complicações clínicas). Nos artigos que trataram as complicações clínicas como exposições de desfechos materno-infantis, as variáveis de caracterização foram: autor, ano de publicação, avaliação da qualidade, objetivos, delineamento, local de estudo, tamanho da amostra, complicações clínicas na gravidez de interesse, desfechos de interesse e principais resultados (complicações clínicas associadas aos desfechos). Os artigos de revisão sistemática foram caracterizados segundo autor, ano de publicação, objetivos, tipos de estudo considerados, abrangência da revisão, número de estudos incluídos na revisão, complicações clínicas na gravidez de interesse e principais resultados.

\section{Avaliação da qualidade dos artigos incluídos}

A avaliação da qualidade metodológica dos estudos empíricos selecionados foi obtida pela adaptação da escala Effective Public Health Practice Project: Quality Assessment Tool for Quantitative Studies - QATQS (http://www.ephpp.ca/ tools.html). Desta escala foram avaliados cinco quesitos (classificados em "fortes", "moderados" ou “fracos"): 1) viés de seleção; 2) desenho de estudo; 3 ) fatores de confundimento; 4) métodos de coleta de dados; e 5) tipo de análise empregada para o desfecho. $\mathrm{O}$ artigo foi classificado de qualidade "forte" quando nenhum dos quesitos foi considerado "fraco"; "moderado", no caso de um dos quesitos ter sido considerado "fraco"; e, "fraco", se dois ou mais quesitos foram avaliados como "fracos". Decidiu-se pela exclusão dos artigos que ficaram na classificação "fraco".

\section{Análise dos dados}

Os artigos empíricos foram agrupados segundo o tratamento dado às complicações clínicas na gravidez (como desfecho ou exposição). Ainda, as análises das complicações clínicas como desfechos foram relatadas em quatro categorias segundo o foco de estudo: (i) transtornos mentais, (ii) doenças infecciosas, (iii) morbidade materna grave e (iv) determinação das principais afecções ou causas de internações. No caso dos estudos em que os pesquisadores analisaram as complicações clínicas como desfecho, os resultados foram sintetizados considerando-se as frequências e os fatores de exposição mais relevantes. Artigos nos quais as complicações clínicas representaram exposição, os resultados sintetizaram-se com base nos desfechos materno-infantis que mostraram associação. Os achados dos artigos de revisão sistemática foram utilizados para fins de comparação com os encontrados nesta revisão.

\section{Resultados}

O fluxograma que apresenta o processo de identificação e seleção dos estudos encontra-se na Figura 1. Foram encontrados 2.984 artigos (620 na BVS, 185 no SciELO e 2.179 no PubMed) publicados a partir de 2010 nos idiomas inglês, português e espanhol nas bases de dados. Dos 2.895 artigos submetidos à triagem, 2.734 foram excluídos (38 referidos a estudos realizados em outros países, 434 sem a inclusão de gestantes e 2.252 que não abordavam complicações clínicas na gravidez). Após a leitura dos títulos e resumos, restaram 161 artigos, em que foram aplicados os critérios de inclusão e exclusão pela leitura dos textos na íntegra. Excluíram-se 120 estudos: seis de revisão não sistemática, seis de cunho qualitativo, três relatos de caso, sete de desenho descritivo, 67 que não utilizaram amostras representa- 


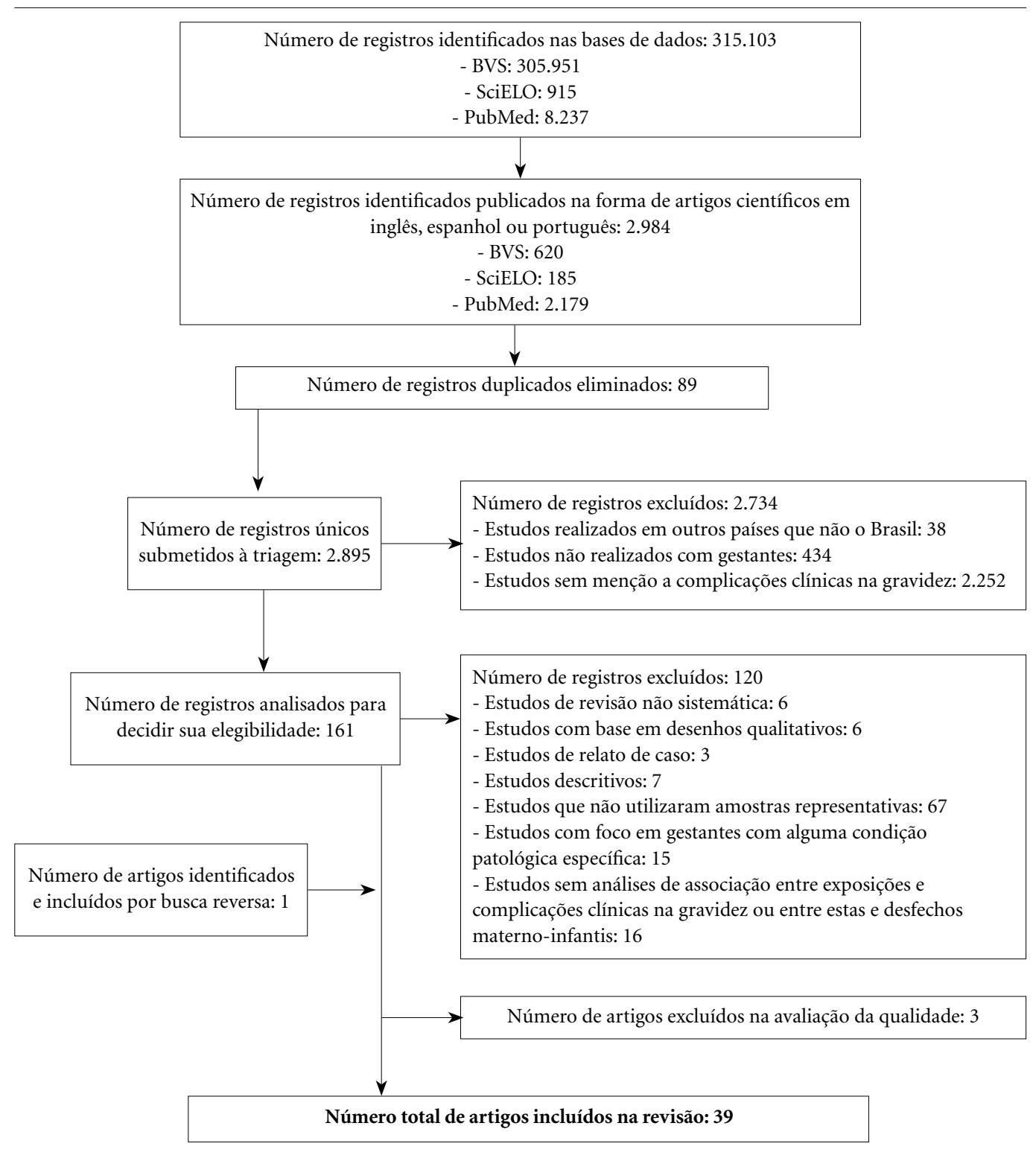

Figura 1. Fluxograma das fases de identificação, triagem e seleção de artigos sobre complicações clínicas em gestantes brasileiras, publicados entre 2010 e 2018.

Fonte: Elaborado pelos autores.

tivas, 15 baseados em gestantes com algum tipo de patologia e 16 sem análises de associação entre exposições e complicações clínicas na gravidez ou entre estas e desfechos materno-infantis. Assim, decidiu-se pela inclusão de 41 artigos, que após a adição de um por busca reversa e a exclusão de três na avaliação da qualidade resultou em 39 artigos incluídos na revisão. O critério de qualidade em que os artigos empíricos apresentaram maior limitação foi, marcadamente, o controle dos fatores de confundimento. Os artigos excluí- dos tiveram classificação "fraco" nos quesitos referidos ao viés de seleção e à análise ajustada da associação entre exposição e desfecho.

A caracterização geral dos estudos empíricos está descrita no Quadro 1, com um total de 30 artigos sobre complicações clínicas na gravidez como desfecho ${ }^{1,4,7,10-36}$, e no Quadro 2, com um total de oito artigos sobre complicações clínicas na gravidez como exposição de desfechos materno-infantis ${ }^{1,27,37-42}$, ressaltando-se que dois artigos apresentaram os dois perfis ${ }^{1,30}$. 


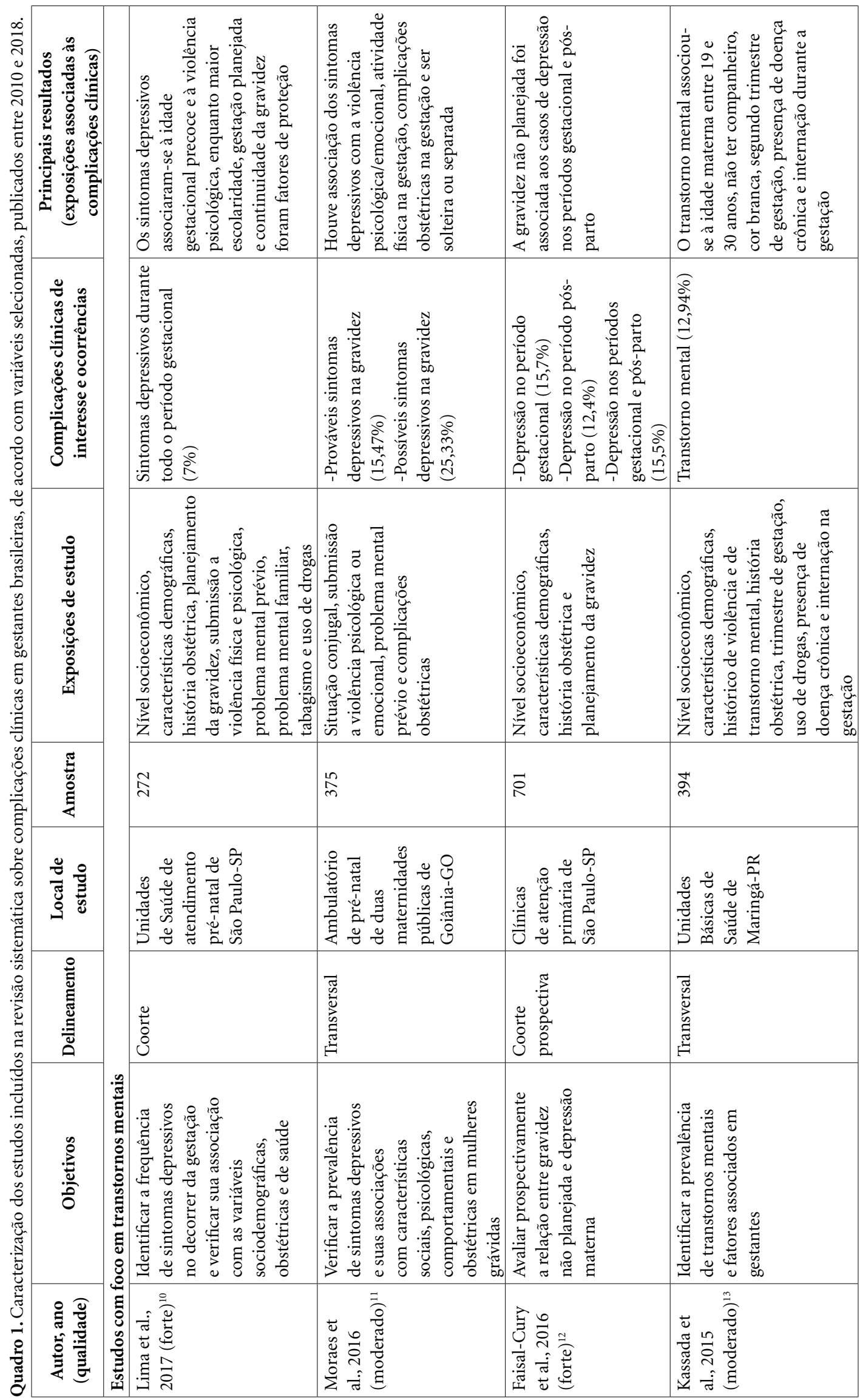




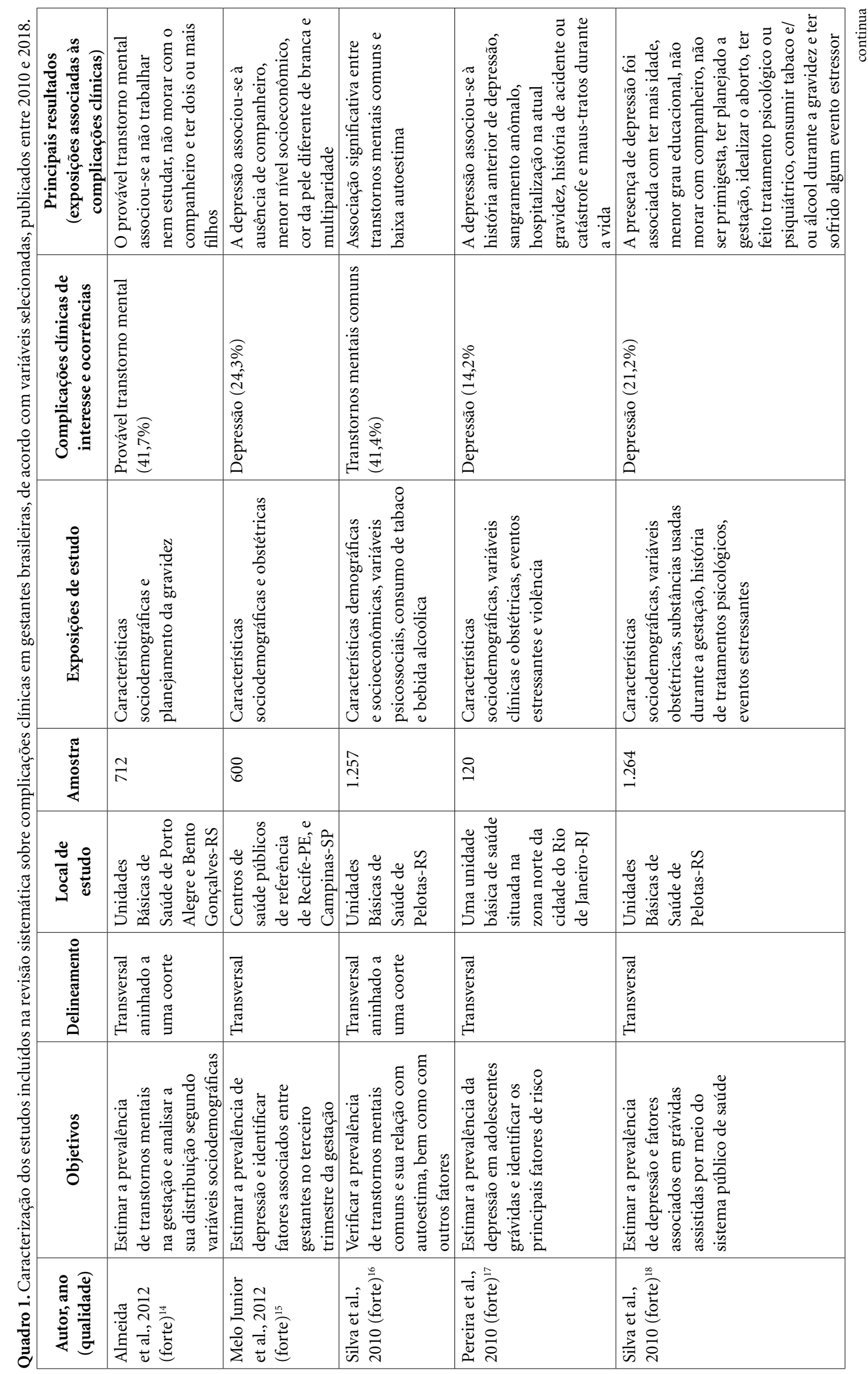




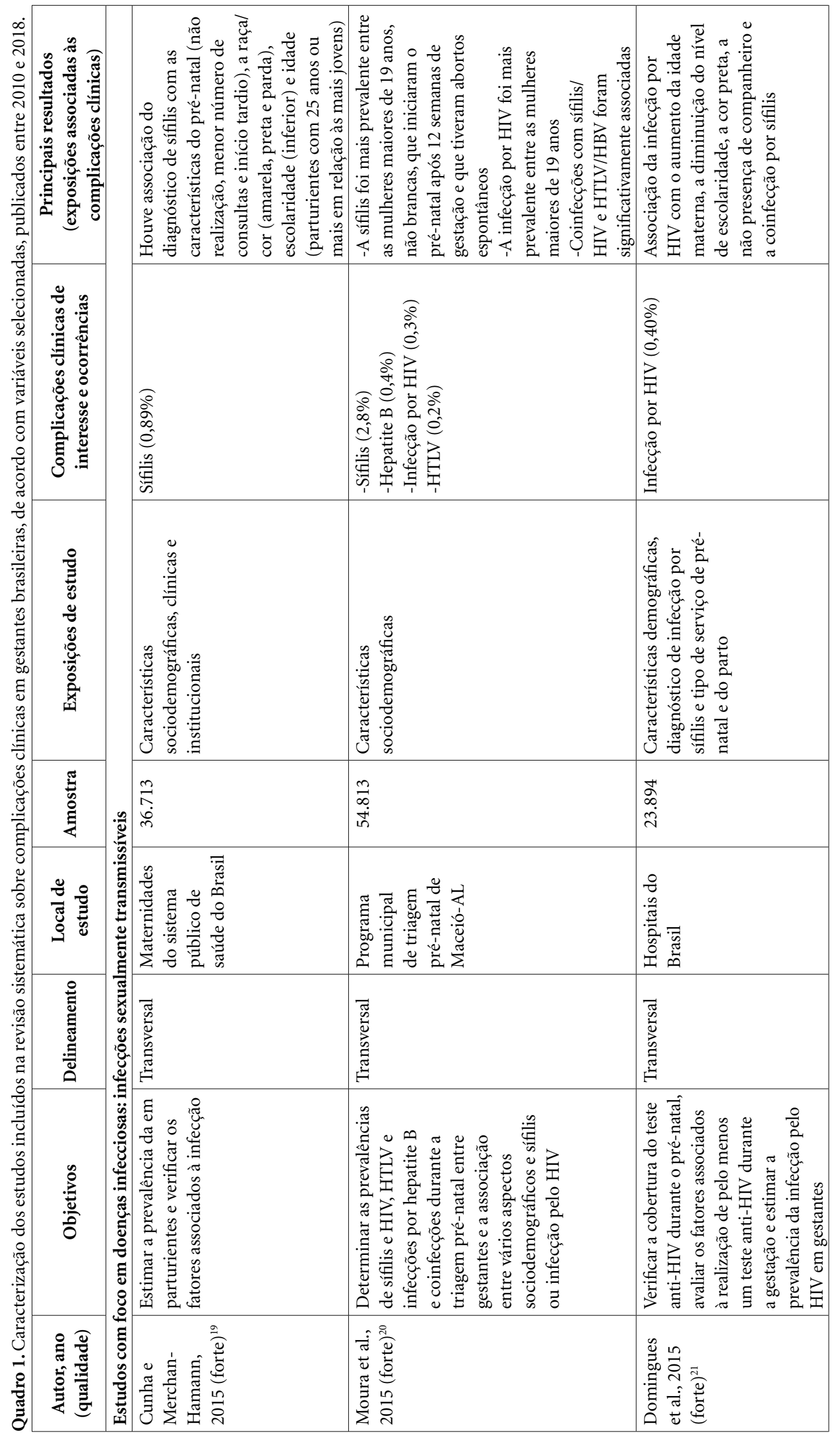




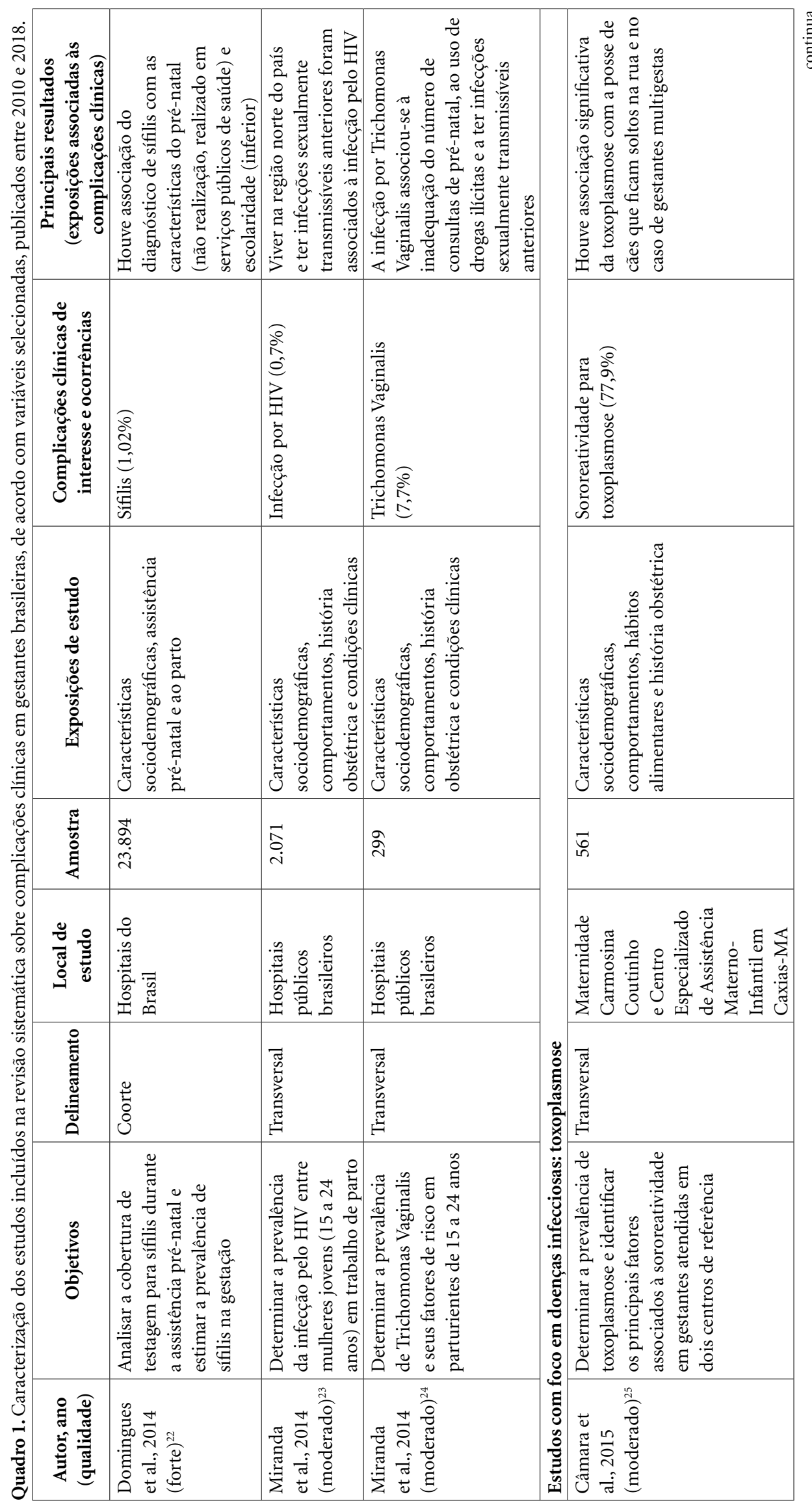




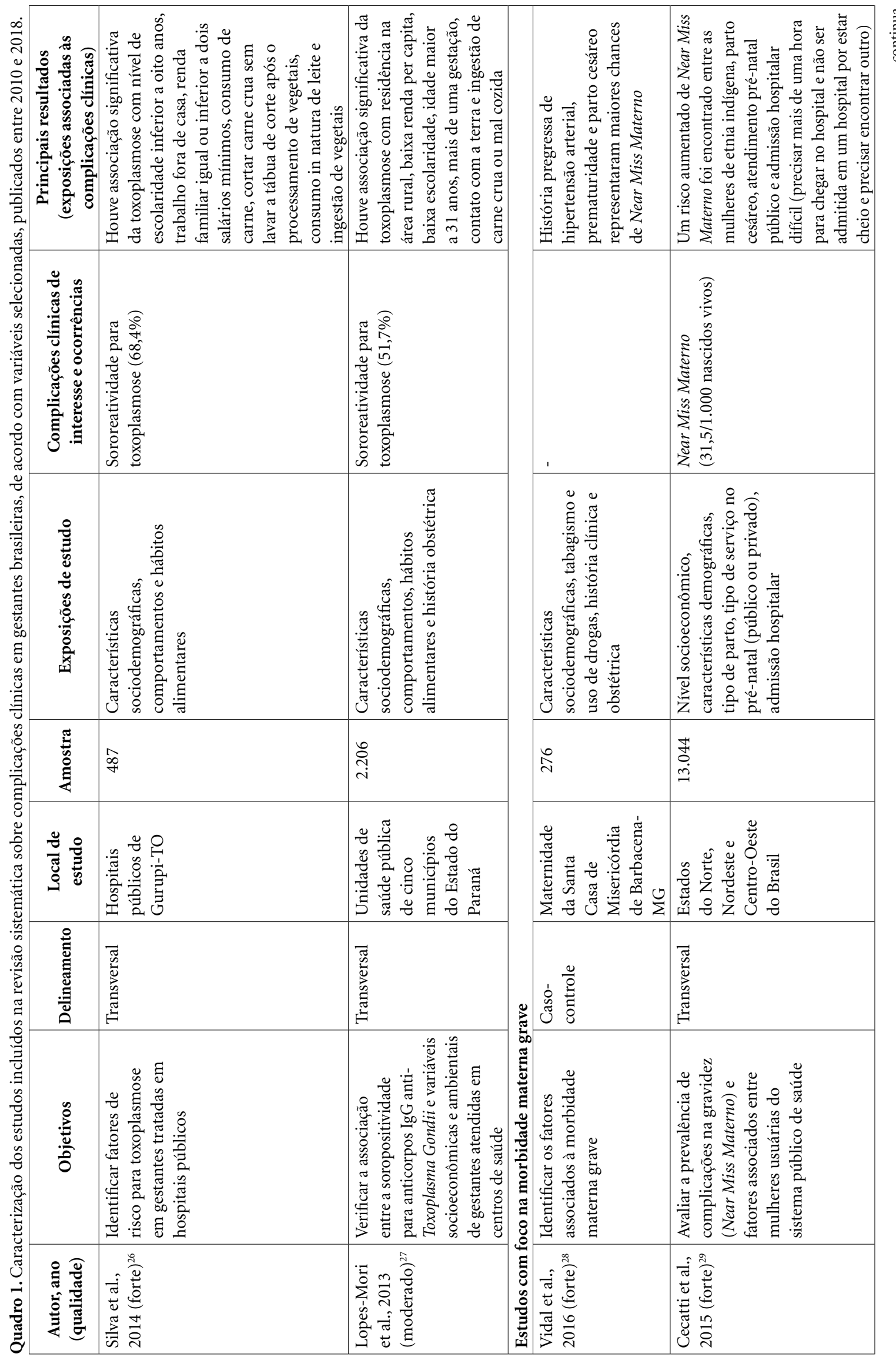




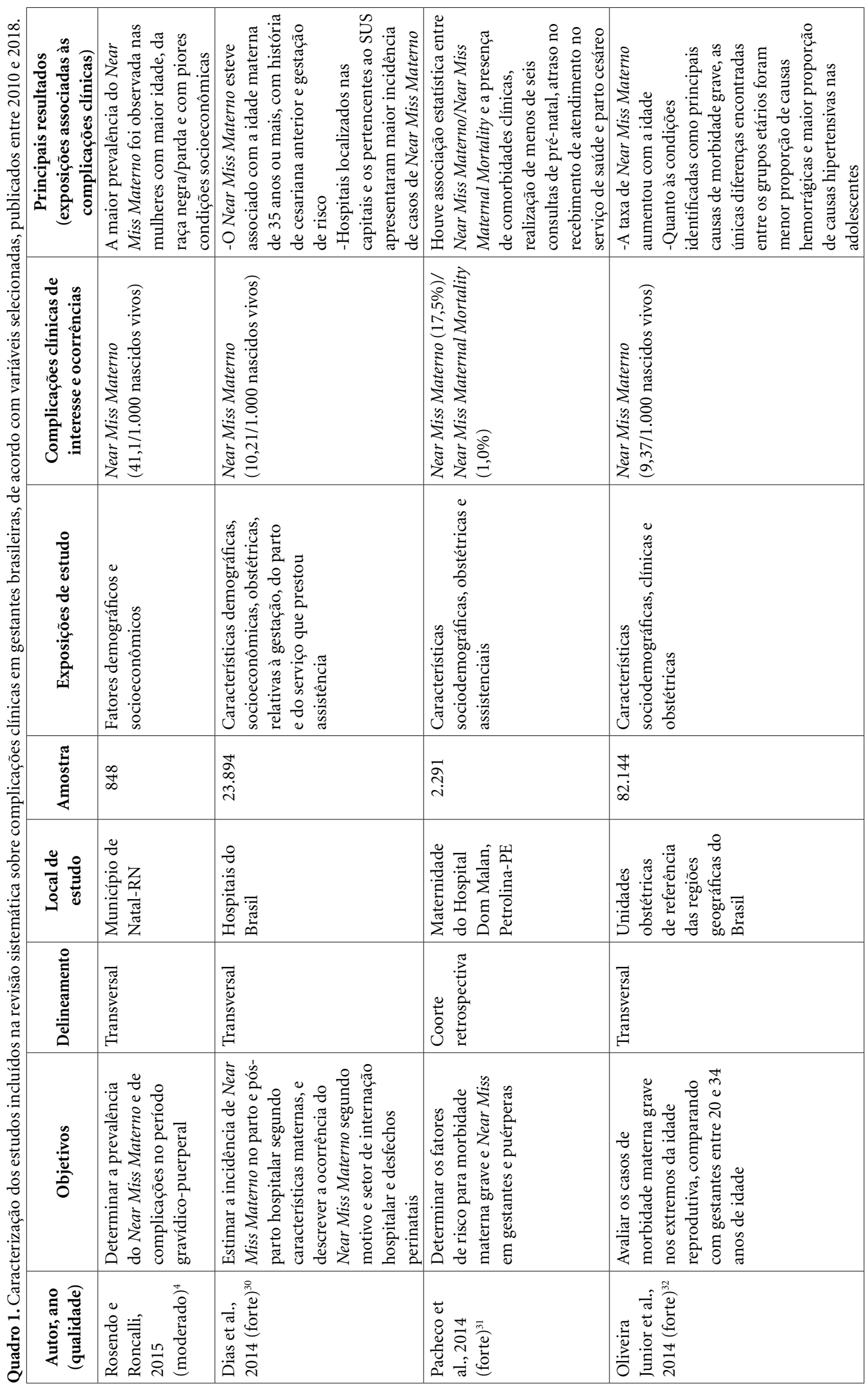




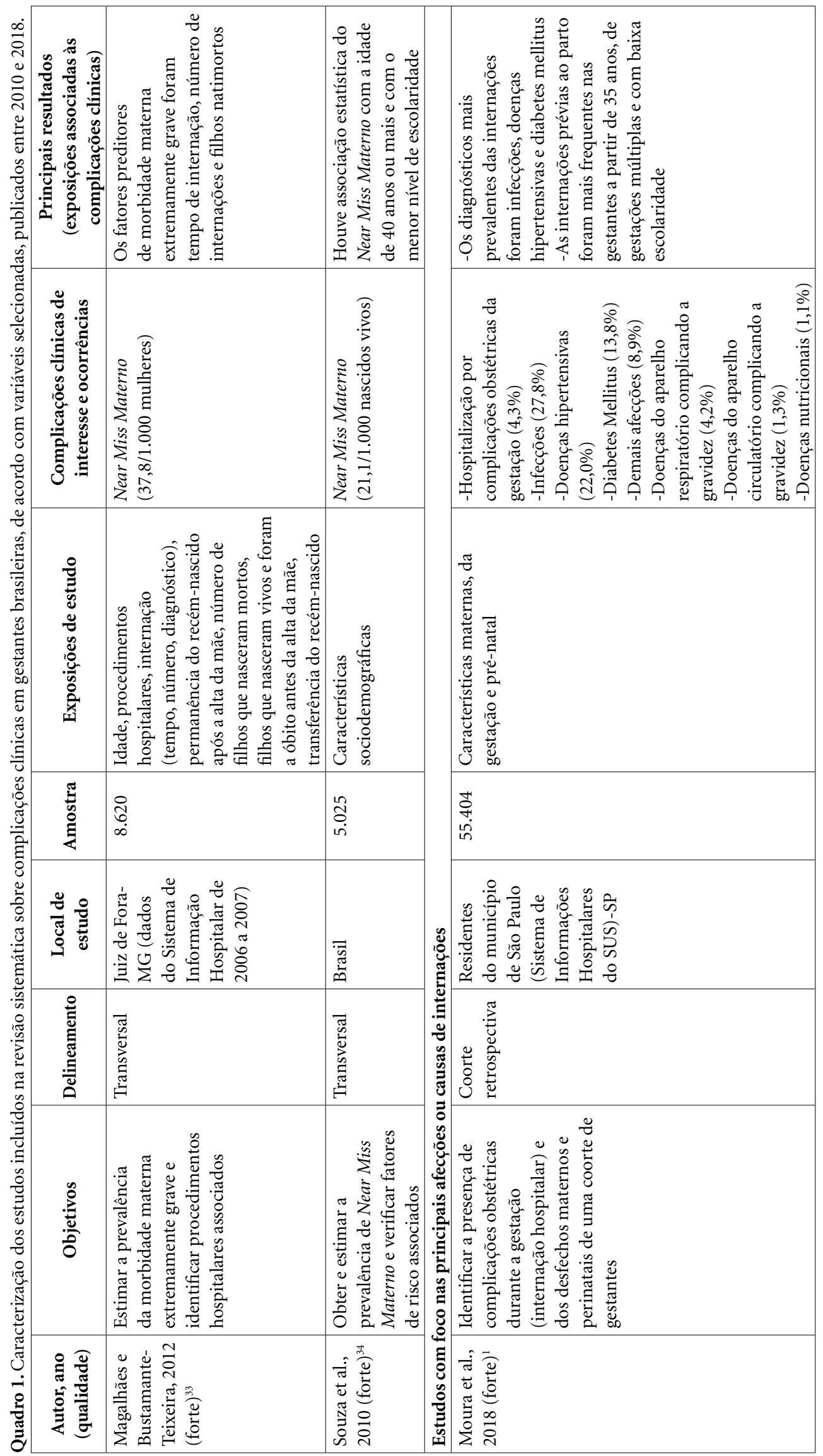




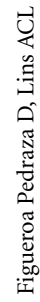

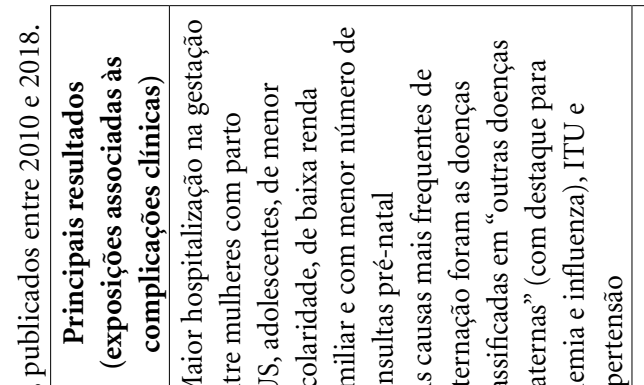

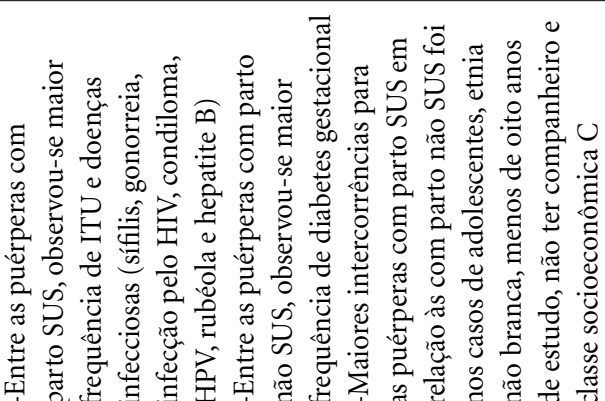

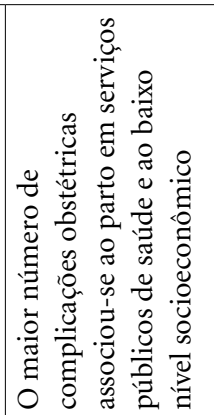

.

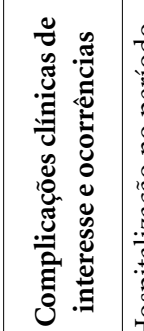

(2)

in

@)

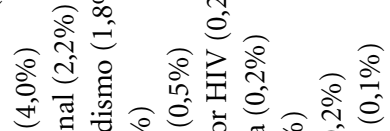

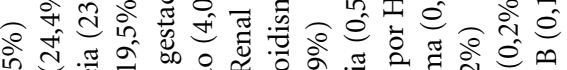
党

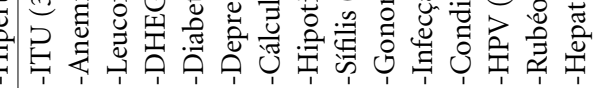

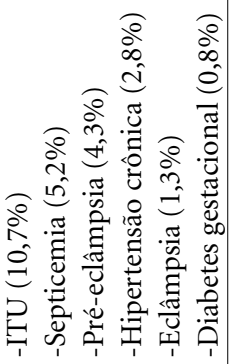

\begin{tabular}{|c|c|c|c|}
\hline 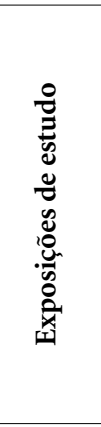 & 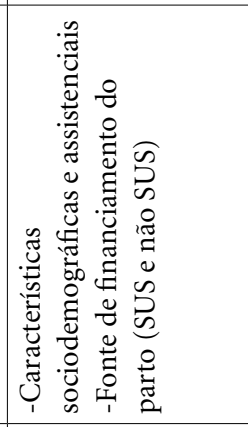 & 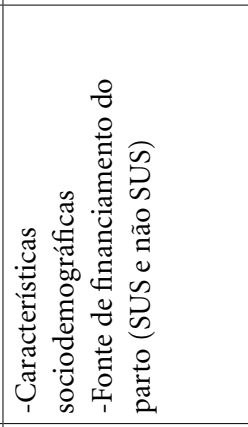 & 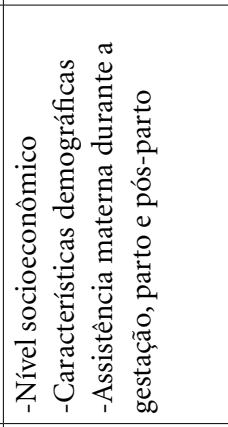 \\
\hline 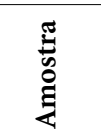 & $\underset{\sim}{\infty}$ & $\stackrel{\infty}{\Omega}$ & $\begin{array}{l}\infty \\
\infty \\
\infty\end{array}$ \\
\hline ఫृ & 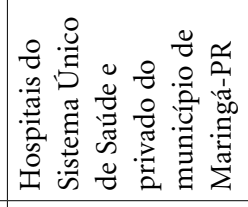 & 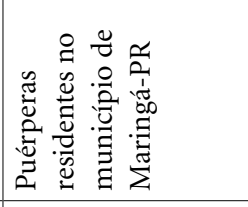 & 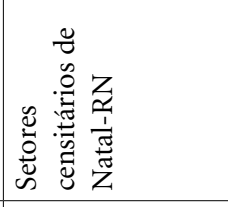 \\
\hline 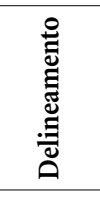 & 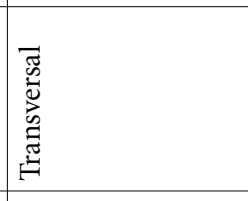 & 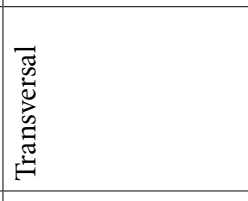 & 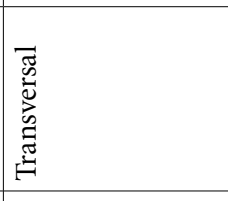 \\
\hline$\frac{2}{0} \cdot \frac{0}{0}$ & 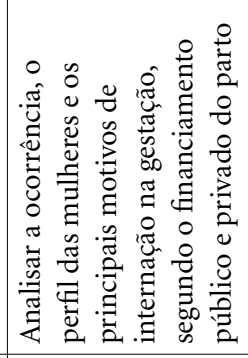 & 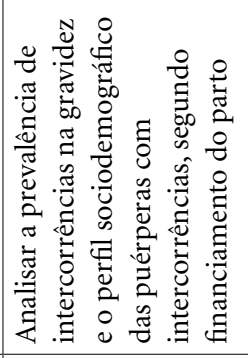 & 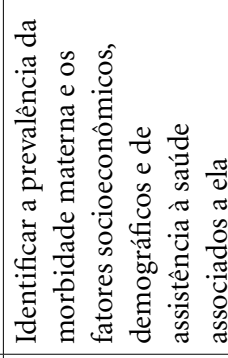 \\
\hline 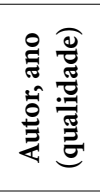 & 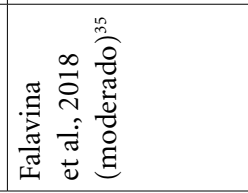 & 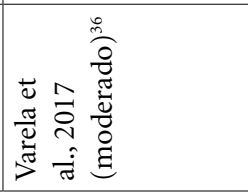 & 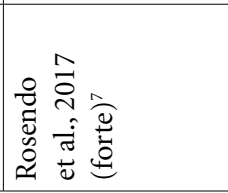 \\
\hline
\end{tabular}




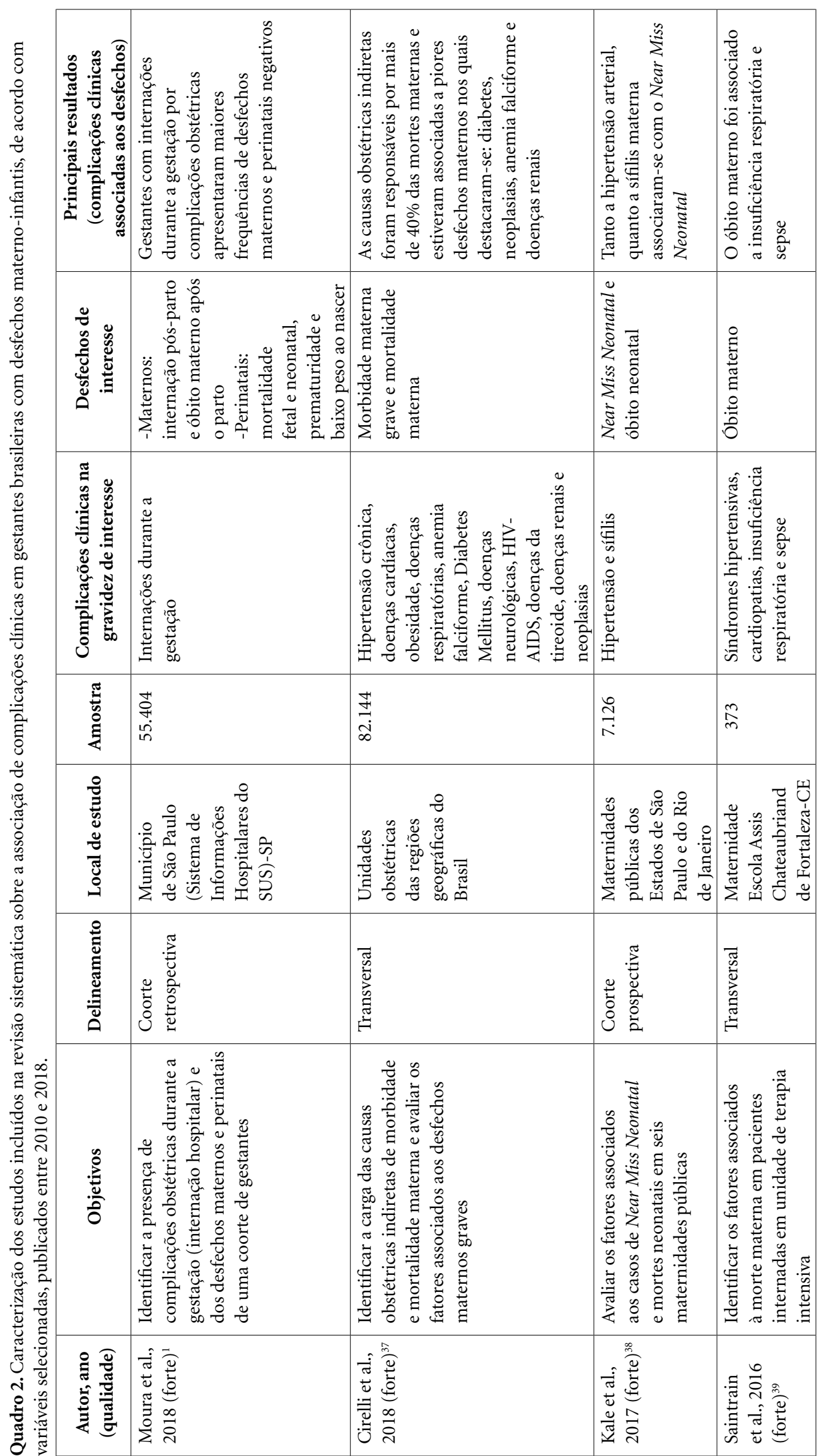




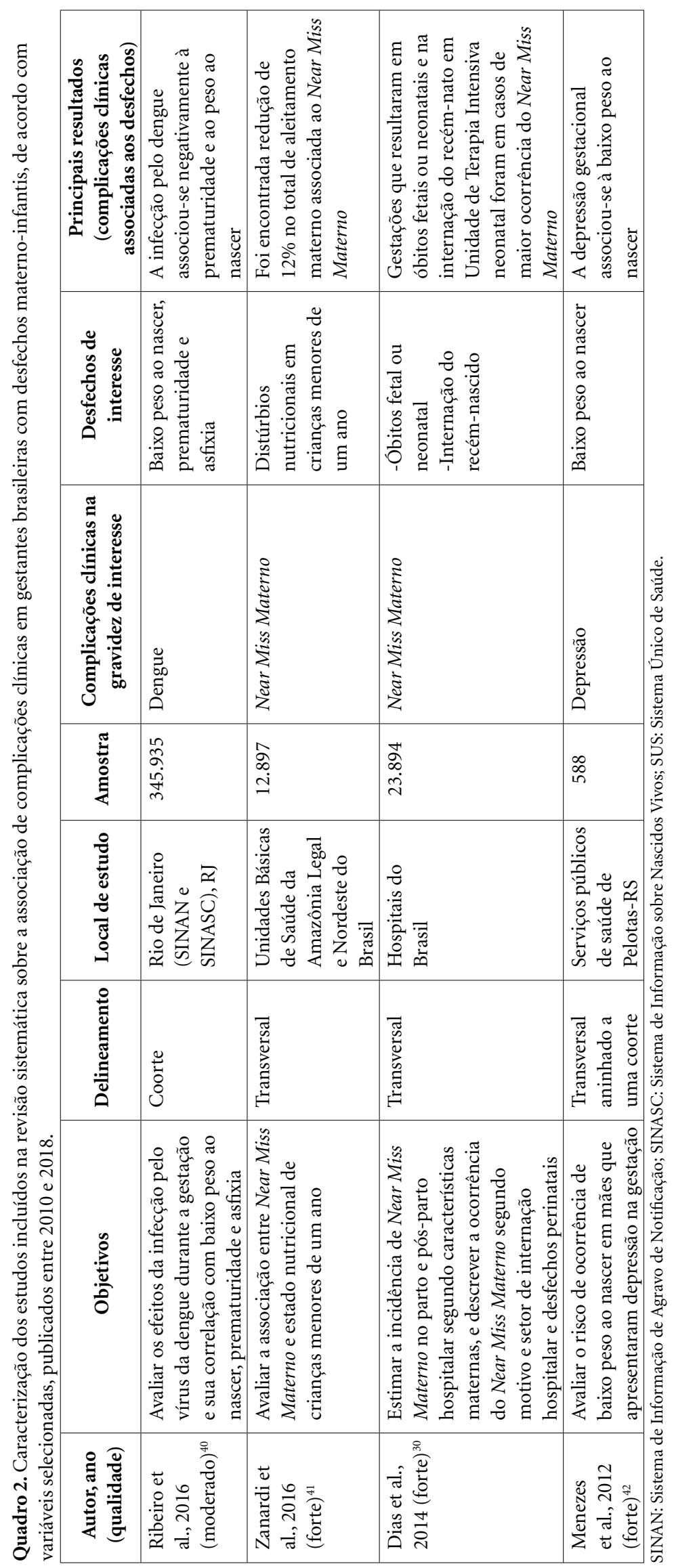

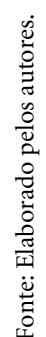


Houve grande diversidade na faixa etária das gestantes pesquisadas, sendo que todos os estudos contemplaram mulheres adolescentes e adultas, excetuando-se um que considerou apenas adolescentes ${ }^{17}$. Em um artigo a informação sobre a idade não foi referida ${ }^{40}$ (dados não apresentados em quadro).

Dos 36 artigos empíricos sistematizados, apenas um é do tipo caso-controle ${ }^{28}$, sete são de coorte ${ }^{1,10,12,22,31,38,40}$ e os outros transversais $^{4,7,11,13-21,23-27,29,30,32-37,39,41}$. Em relação ao local de realização dos estudos, destaca-se que 11 deles são de âmbito nacional ${ }^{19,21-24,30,32,34,37}$ ou regional ${ }^{29,41}$. Os outros foram realizados em localidades das regiões Sudeste ${ }^{1,10,12,15,17,28,33,38,40}$, Sul $^{13,14,16,18,27,35,36,42}$, Nordeste $^{4,7,15,20,25,31,39}$, Centro-Oeste ${ }^{11}$ e Norte ${ }^{26}$, ressaltando-se que um estudo compreendeu cidades do Sudeste e Nordeste ${ }^{15}$. O tamanho da amostra variou de $272^{10}$ a $345.935^{40}$, excluindo-se um estudo que foi desenvolvido apenas com adolescentes, do qual participaram 120 indivíduos $^{17}$ (Quadros 1 e 2).

No que se refere às complicações clínicas analisadas como desfechos (Quadro 1), nove estudos referiram-se a transtornos mentais ${ }^{10-18}$, nove a doenças infecciosas ${ }^{19-27}$ e oito à morbidade materna grave $e^{4,28-34}$. Ainda, quatro artigos tiveram como foco a determinação das principais afecções ou causas de internaçãoo, $1,73,36$. No caso das doenças infecciosas, seis artigos trataram sobre infecções sexualmente transmissíveis ${ }^{19-24}$ e três sobre toxoplasmose $\mathrm{e}^{25-27}$.

As prevalências de problemas mentais oscilaram entre $7 \%{ }^{10} \mathrm{e} 41,7 \%{ }^{14}$. A não convivência com companheiro ${ }^{11,13-15,18}$, a violência psicológica/ emocional/estresse $\mathrm{e}^{10,11,17,18}$, a presença de complicações clínicas na gestação ${ }^{11,13,17,18} \mathrm{e}$ a multiparidade ${ }^{14,15,18}$ associaram-se às doenças psicológicas em mais de dois dos artigos.

Infecções sexualmente transmissíveis apresentaram prevalências baixas ${ }^{19-23}$, a exceto da infecção por Trichomonas Vaginalis de $7,7 \%{ }^{24}$, associadas, principalmente, à maior idade ${ }^{19-21}$, menor escolaridade ${ }^{19,21,22}$, pré-natal inadequado ${ }^{19,20,22,24} \mathrm{e}$ características raciais $^{19-21}$. Além disso, a coinfecção/infecção prévia também foi apontada como fator importante ${ }^{20,21,24}$.

A ocorrência de toxoplasmose variou de $51,7 \%{ }^{27}$ a $77,9 \%{ }^{25}$ e teve como principais exposições relacionadas o consumo de carne ${ }^{26,27}$, a baixa escolaridade ${ }^{26,27}$, a renda insuficiente ${ }^{26,27}$ e não ser a primeira gestação ${ }^{25,27}$.

$\mathrm{O}$ indicador de morbidade materna grave Near Miss Materno teve como valores menor e maior 9,37/1.000 nascidos vivos ${ }^{32}$ e 41,1/1.000 nascidos vivos ${ }^{4}$, respectivamente. Parto cesáreo $^{28-31}$, maior idade ${ }^{4,30,32,34}$, problemas nos atendimentos de saúde ${ }^{29,31}$, uso de serviços públicos de saúde ${ }^{29,30}$ e características raciais ${ }^{4,29}$ representaram os principais fatores que interferiram na morbidade materna grave.

Por meio dos estudos com foco nas principais afecções ou causas de internação na gestação, têm-se como complicações citadas em no mínimo dois artigos e com frequências superiores a 10\% a Infecção do Trato Urinário ${ }^{7,35,36}$, a ane$\mathrm{mia}^{35,36} \mathrm{e}$ as doenças hipertensivas ${ }^{1,36}$. A análise de fatores associados às hospitalizações indica a influência da idade materna nos extremos reprodutivos ${ }^{1,35,36}$, da baixa escolaridade ${ }^{1,35}$, da condição socioeconômica ${ }^{7,35,36}$ e do uso dos serviços públicos de saúde d,35,36. $^{7}$.

Nos artigos em que pesquisadores mediram desfechos materno-infantis (Quadro 2) 1,30,37-42, observa-se o uso de marcadores variados: morbidade grave ${ }^{37,38}$, internação pós-parto ${ }^{1,30}$, óbito $^{1,30,37-39} \mathrm{e}$ indicadores da saúde infantil (prematuridade, baixo peso ao nascer $)^{1,40-42}$. Em todos os estudos foi possível observar associação entre as complicações clínicas na gravidez e os desfechos de interesse, exceto em um artigo no qual a mortalidade foi reportada como não associada à hipertensão arterial e sífilis materna ${ }^{33}$.

Os estudos de revisão sistemática incluí$\operatorname{dos}^{43-45}$ estão caracterizados no Quadro 3. Os artigos tiveram como foco de estudo o near miss materno em gestantes brasileiras ${ }^{43}$, o risco associado à dengue durante a gravidez em quatro desfechos fetais adversos ${ }^{44} \mathrm{e}$ a avaliação das complicações clínicas na gestação de adolescentes ${ }^{45}$. Os resultados destacaram a associação do near miss materno com o desenvolvimento de doenças hipertensivas e hemorragia ${ }^{43}$, o risco de natimortalidade, aborto espontâneo, prematuridade e baixo peso ao nascer associado à dengue sintomática na gravidez ${ }^{44}$, e doenças hipertensivas, síndromes hemorrágicas, infecção urinária, prematuridade, baixo peso ao nascer e mortalidade perinatal como principais problemas da gravidez na adolescência ${ }^{45}$.

\section{Discussão}

Foram identificados nesta revisão 36 artigos de base empírica que abordaram as complicações clínicas na gravidez no Brasil, dos quais um número elevado foi conduzido em âmbito nacional e todas as regiões geográficas foram representadas. No perímetro regional, destacou-se o Sudeste, Sul 
Quadro 3. Caracterização dos estudos incluídos na revisão sistemática que sistematizaram a produção científica sobre complicações clínicas na gravidez incluindo gestantes brasileiras, de acordo com variáveis selecionadas, publicados entre 2010 e 2018.

\begin{tabular}{|c|c|c|c|c|c|c|}
\hline $\begin{array}{l}\text { Autor, } \\
\text { ano }\end{array}$ & Objetivos & $\begin{array}{c}\text { Tipos de } \\
\text { estudo } \\
\text { considerados }\end{array}$ & Abrangência & $\begin{array}{l}\text { Número } \\
\text { de estudos } \\
\text { incluídos }\end{array}$ & $\begin{array}{c}\text { Complicações } \\
\text { clínicas na } \\
\text { gravidez de } \\
\text { interesse }\end{array}$ & Principais resultados \\
\hline $\begin{array}{l}\text { Silva et } \\
\text { al., } 2018^{43}\end{array}$ & $\begin{array}{l}\text { Analisar a } \\
\text { frequência, } \\
\text { características } \\
\text { e causas da } \\
\text { morbidade } \\
\text { materna grave } \\
(\text { Near Miss } \\
\text { Materno) no } \\
\text { Brasil }\end{array}$ & $\begin{array}{l}\text { Estudos } \\
\text { descritivos e } \\
\text { de associação } \\
\text { (transversais } \\
\text { ou } \\
\text { longitudinais) }\end{array}$ & Brasil & 48 & $\begin{array}{l}\text { Near Miss } \\
\text { Materno }\end{array}$ & $\begin{array}{l}\text {-A Razão de Near Miss } \\
\text { Materno variou de } \\
2,4 / 1.000 \text { nascidos vivos } \\
\text { a } 188,4 / 1.000 \text { nascidos } \\
\text { vivos } \\
\text {-Doenças hipertensivas } \\
\text { e hemorragia foram } \\
\text { as morbidades mais } \\
\text { comuns } \\
\text {-Complicações clínicas } \\
\text { (hemorragia, hipertensão } \\
\text { prévia), falhas nos } \\
\text { cuidados de saúde } \\
\text { (associação protetora } \\
\text { do pré-natal), fatores } \\
\text { sociodemográficos (cor } \\
\text { da pele não branca, } \\
\text { adolescência/idade } \geq 35 \\
\text { anos, baixa escolaridade), } \\
\text { parto cesáreo atual ou } \\
\text { prévio e aborto prévio } \\
\text { foram associadas ao Near } \\
\text { Miss Materno }\end{array}$ \\
\hline $\begin{array}{l}\text { Paixão et } \\
\text { al., } 2016^{44}\end{array}$ & $\begin{array}{l}\text { Estimar o risco } \\
\text { de natimorta- } \\
\text { lidade, aborto } \\
\text { espontâneo, } \\
\text { parto prema- } \\
\text { turo e baixo } \\
\text { peso ao nascer } \\
\text { em mulheres } \\
\text { que tiveram } \\
\text { infecção por } \\
\text { dengue duran- } \\
\text { te a gravidez }\end{array}$ & $\begin{array}{l}\text { Estudos de } \\
\text { caso-controle, } \\
\text { coorte, } \\
\text { transversal e } \\
\text { séries de casos }\end{array}$ & $\begin{array}{l}\text { Mundial, } \\
\text { incluindo o } \\
\text { Brasil }\end{array}$ & $\begin{array}{l}16 \text { (dois } \\
\text { desenvolvidos } \\
\text { no Brasil) }\end{array}$ & Dengue & $\begin{array}{l}\text {-Nascimento prematuro } \\
\text { e baixo peso ao nascer } \\
\text { foram os desfechos } \\
\text { adversos mais comuns da } \\
\text { gravidez } \\
\text {-As análises sugerem } \\
\text { associação entre a dengue } \\
\text { sintomática na gravidez } \\
\text { e cada um dos quatro } \\
\text { desfechos fetais adversos } \\
\text { (natimortalidade, aborto } \\
\text { espontâneo, parto } \\
\text { prematuro e baixo peso } \\
\text { ao nascer) }\end{array}$ \\
\hline $\begin{array}{l}\text { Azevedo } \\
\text { et al., } \\
2015^{45}\end{array}$ & $\begin{array}{l}\text { Avaliar as } \\
\text { complicações } \\
\text { relacionadas } \\
\text { à gravidez na } \\
\text { adolescência }\end{array}$ & $\begin{array}{l}\text { Ensaios } \\
\text { clínicos, } \\
\text { estudos } \\
\text { observacionais } \\
\text { analíticos } \\
\text { (caso- } \\
\text { controle, } \\
\text { coorte } \\
\text { prospectivos e } \\
\text { retrospectivos) } \\
\text { e estudos } \\
\text { descritivos } \\
\text { transversais }\end{array}$ & $\begin{array}{l}\text { Mundial, } \\
\text { incluindo o } \\
\text { Brasil }\end{array}$ & $\begin{array}{l}15 \text { (seis } \\
\text { desenvolvidos } \\
\text { no Brasil) }\end{array}$ & $\begin{array}{l}\text { Complicações } \\
\text { relacionadas } \\
\text { à gravidez na } \\
\text { adolescência }\end{array}$ & $\begin{array}{l}\text { As complicações clínicas } \\
\text { maternas mais descritas } \\
\text { nos estudos, incluindo } \\
\text { os desenvolvidos no } \\
\text { Brasil, foram as doenças } \\
\text { hipertensivas, as } \\
\text { síndromes hemorrágicas } \\
\text { e a infecção urinária; } \\
\text { os principias desfechos } \\
\text { relatados foram } \\
\text { prematuridade, baixo } \\
\text { peso ao nascer e } \\
\text { mortalidade perinatal }\end{array}$ \\
\hline
\end{tabular}

Fonte: Elaborado pelos autores. 
e Nordeste, pelo quantitativo de estudos realizados, assemelhando ao observado em outras revisões com foco na morbidade materno-infantil ${ }^{43,46}$.

Segundo os resultados sistematizados, os transtornos mentais, as doenças infecciosas e a morbidade materna grave foram as principais complicações clínicas tratadas pelos autores nos seus artigos. Ainda, houve interesse pela análise das principais afecções ou causas de internação na gravidez.

A condição psicológica da mãe durante a gestação constitui o resultado de variações endócrinas complexas ${ }^{11}$. Os sintomas depressivos na gravidez $^{10,11}$, depressão gestacional ${ }^{12,15,18}$ e transtorno mental ${ }^{13,14,16}$ apresentaram-se prevalentes em estudos sistematizados, associando-se, principalmente, à não convivência com companheiro ${ }^{11,13-15,18}$, a complicações obstétricas, internação ou presença de doença crônica na gestação ${ }^{11,13,17,18}$, à violência psicológica/emocional ${ }^{10,11,17,18} \mathrm{e}$ à multiparidade $^{14,15,18}$. Esses resultados condizem com os de pesquisas de grande abrangência que identificaram os transtornos mentais na gestação associados à gestação multípara, na Holanda ${ }^{47}$, à condição materna solteira/separada e à presença de doença crônica, em populações multiétni$\operatorname{cas}^{48}$, a complicações obstétricas, na região sul de Israel $^{49}$ e à violência psicológica/emocional, em Província do Norte de Ruanda e na cidade de Kigali ${ }^{50}$. Esses fatores colocam as gestantes em vulnerabilidade dado que propiciam desfechos adversos como intercorrências clínicas, depressão puerperal, psicose puerperal, mortalidade infantil e déficit no desenvolvimento do bebê $\hat{~}^{13}$.

As infecções sexualmente transmissíveis constituem as principais ameaças à saúde, atingindo populações em diversas partes do mundo, com maior destaque para os países de baixa renda. As mulheres, por serem fisiologicamente mais vulneráveis, são ainda mais afetadas. Dessa forma, o risco aumentado durante o período gestacional pode desencadear graves complicações ao recémnascido ${ }^{51}$. Nesta revisão, as infecções por Trichomonas Vaginalis foram as mais prevalentes nos estudos incluídos. Resultado semelhante foi observado em revisão sobre a prevalência de infecções sexualmente transmissíveis entre mulheres grávidas de 30 países de baixa e média renda, no qual a infecção por Trichomonas Vaginalis variou de 3,9\%, na América Latina, a 24,6\%, na África Austral $^{52}$. A Trichomonas Vaginalis configura a infecção sexualmente transmissível curável mais comum em todo o mundo, com prevalência superior entre as mulheres e forte associação com a saúde e desvantagem social ${ }^{53}$.
Globalmente, a sífilis materna é a infecção mais comumente associada à ocorrência de fetos natimortos em populações menos favorecidas economicamente. Essa infecção quando não tratada resulta em sífilis congênita em cerca de $16 \%$ dos casos, além de estar associada a 90.000 óbitos neonatais e 65.000 recém-nascidos prematuros ou com baixo peso ao nascer ${ }^{54}$. Apesar das baixas prevalências encontradas na revisão atual, a sífilis ${ }^{19,20,22}$ e o HIV ${ }^{20,21,23}$ foram as infecções sexualmente transmissíveis discutidas em um maior número de estudos.

Características como raça/cor não branca, baixo nível de escolaridade, maior idade e baixa qualidade do pré-natal que se mostraram estatisticamente associadas a infecções sexualmente transmissíveis em outros inquéritos com gestantes brasileiras, cujos resultados foram divulgados recentemente ${ }^{55,56}$, corroboram-se por meio desta revisão. Essas características impõem desigualdades sociais que implicam na necessidade de medidas com foco na prevenção, orientação e gerenciamento de risco para infecções sexualmente transmissíveis, com a participação de profissionais de saúde e a comunidade ${ }^{55}$.

Os resultados de pesquisas desenvolvidas em outros países corroboram os encontrados na presente revisão para a maioria dos fatores associados à ocorrência de infecções por toxoplasmose. Um estudo realizado com populações multiétnicas encontrou a presença de anticorpos associadas a hábitos alimentares como o consumo de carne ${ }^{57}$. As consequências dos maus hábitos de lavagem das mãos e a higiene inadequada dos utensílios domésticos após o contato com a carne crua levam ao risco de ingestão de cistos de bradizito presentes em alimentos contaminados. A associação do Toxoplasma Gondii com a baixa escolarida$\mathrm{de}^{26,27}$ e não ser a primeira gestação $\mathrm{O}^{25,27}$ observada na presente revisão foi semelhante entre mulheres grávidas do Marrocos $^{58}$. Cabe ressaltar que a baixa escolaridade está relacionada a condições de vida que implicam em maior risco de contaminação, visto que uma melhor educação reduz a exposição devido à adoção de hábitos de higiene mais adequados, principalmente em relação ao manuseio de alimentos ${ }^{26}$. A associação com a multiparidade pode ser explicada pelo aumento da exposição a fontes de infecção ao longo da vida ${ }^{58}$. Entretanto, a renda insuficiente que mostrou associação à toxoplasmose em dois dos estudos incluídos nessa revisão ${ }^{26,27}$ contraria os resultados de revisões sistemáticas considerando pesquisas desenvolvidas com mulheres em idade reprodutiva e gestantes de Arábia Saudita ${ }^{59}$ e Irão ${ }^{60}$. A soroprevalência 
do Toxoplasma Gondii varia amplamente em diferentes partes do mundo ${ }^{57}$. Nessa conjuntura, é fundamental que cada país possua informações epidemiológicas peculiares, a fim de elaborar estratégias que atendam a cada realidade ${ }^{25}$, o que denota a importância dos atuais achados.

A morbidade materna grave ou near miss materno constitui uma condição de quase morte, seguida de sobrevivência a uma complicação materna grave, ocorrida durante a gravidez, o parto ou puerpério. Além do comprometimento à saúde materna, essa circunstância tem importante impacto sobre os desfechos fetais e neonatais ${ }^{43}$. Os resultados da presente revisão apontaram entre os principais fatores associados ao near miss materno/morbidade materna grave, a maior idade materna ${ }^{4,30}$ e o parto cesariana ${ }^{28-31}$ assemelhando-se aos de revisão especificamente sobre este desfecho ${ }^{43}$. Em diversas situações a indicação do parto cesariana possivelmente ocorre por alguma complicação materna ocorrida durante o período gestacional e/ou parto, sendo considerada a causa propriamente dita do near miss materno ${ }^{30}$. No que diz respeito à idade materna, cabe destacar que a maior idade nesse período é considerada alto risco para complicações obstétricas em virtude de uma maior prevalência de comorbidades e/ou multiparidade ${ }^{4}$.

Além disso, o uso do serviço público de saúde ${ }^{29-31}$ também foi um fator importante na determinação do near miss materno, semelhante ao observado em um estudo multicêntrico conduzido em 27 centros obstétricos de todas as regiões brasileiras, o qual distinguiu o atraso na prestação de cuidados de saúde obstétrica pelo serviço público de saúde como associado à morbidade materna grave $^{61}$. Essa relação decorre, provavelmente, do maior risco reprodutivo das gestantes atendidas no setor público. Ainda, os serviços públicos de referência materna e neonatal se responsabilizam pela assistência à parte das gestantes de alto risco do Sistema de Saúde Suplementar, quando não há a assistência necessária à situação de risco pela cobertura assistencial ${ }^{30}$.

A característica racial foi outro fator relevante na determinação do near miss materno apontado por essa revisão ${ }^{4,29}$, sendo encontrado resultado similar em estudo prévio sobre disparidades raciais, que registrou maior prevalência nas mulheres negras ${ }^{62}$. A raça/etnia constitui uma das principais formas de se avaliar as iniquidades em saúde nesse campo, por ser considerado um indicador das condições socioeconômicas ${ }^{4}$, a exemplo das populações indígenas que têm inadequado acesso a programas de prevenção e pro- moção da saúde, como campanhas de vacinação, programas de planejamento familiar e políticas de atenção à saúde materna ${ }^{29}$.

A internação hospitalar configura uma intervenção que repercute negativamente durante a gravidez, expondo a gestante e sua família a situação de vulnerabilidade ${ }^{35}$. A Infecção do Trato Urinário ${ }^{7,35,36}$, a anemia ${ }^{35,36} \mathrm{e}$ as doenças hipertensivas ${ }^{1,36}$ identificadas nessa revisão como principais causas de internações na gestação foram destacadas de maneira similar em estudos realizados na França ${ }^{63,64}$. Nos EUA, os achados convergem em relação aos problemas de hipertensão e do trato urinário ${ }^{65}$, constatado também numa revisão da literatura com foco em gestantes adolescentes $^{45}$. Ainda, ao respeito das doenças hipertensivas nas gestantes brasileiras cabe ressaltar que representam uma das morbidades mais comuns relacionadas ao near miss materno ${ }^{43}$ que contribuem com a morbidade materna grave e mortalidade materna em cerca de $70 \%$ e $10,7 \%$ dos casos, respectivamente ${ }^{4}$. Assim, recomendase que anemia, infecção urinária e hipertensão devem estar entre os alvos principais das medidas de prevenção e tratamento para evitar internações durante a gestação ${ }^{35}$.

Estudo de base populacional em gestantes realizado na Dinamarca identificou os extremos de idade reprodutiva da mulher, o baixo nível educacional e o desemprego como fatores relacionados à hospitalização $0^{66}$, resultado mostrado também por meio da atual pesquisa. Gestantes em idades extremas do ponto de vista reprodutivo são mais propícias a apresentarem intercorrências que levam as internações hospitalares tendo em vista que a idade materna avançada está relacionada ao surgimento de doenças crônicas, enquanto na adolescência há maior incidência de complicações na gravidez (síndrome hipertensiva, anemia, diabetes) e no parto ${ }^{1,45}$. A baixa escolaridade materna pode representar aumento do risco de hospitalizações pré-natais por representar indiretamente privações econômicas que interferem no estado de saúde da gestante e a dificuldade de compreender e seguir as orientações educativas, o que explica também a determinação da situação socioeconômica ${ }^{1}$. Mulheres que usam os serviços públicos de saúde apresentam também vulnerabilidade social e econômica relacionadas às iniquidades em saúde que as colocam, também, em maiores probabilidades de internação hospi$\operatorname{talar}^{35}$, desenhando o panorama representado em artigos sistematizados nesse estudo ${ }^{7,35,36}$.

As complicações relacionadas à gravidez, parto e puerpério constituem fatores determinantes 
para desfechos desfavoráveis, evidenciando um importante problema de saúde nos países em desenvolvimento e/ou subdesenvolvidos ${ }^{28}$, como mostrado para as gestantes brasileiras segundo os estudos incluídos nessa revisão $\mathrm{O}^{1,30,37-42}$. Outras revisões da literatura também registraram a relação de eventos adversos, como prematuridade, baixo peso ao nascer e mortalidade perinatal, com as condições clínicas indesejáveis durante o período gestacional $^{44,45,67}$. Essas intercorrências configuram um indicador fundamental para a avaliação da situação de saúde da mulher na comunidade, que possibilite a antecipação a desfechos desfavoráveis ${ }^{1,68}$, refletindo a falta de acesso aos serviços de saúde e as desigualdades sociais ${ }^{2,41}$. Assim, um modelo de atenção à saúde que disponha de acesso ao planejamento familiar e à assistência obstétrica durante o ciclo gravídico-puerperal é fundamental para reduzir essa situação ${ }^{7}$. O pré-natal quando realizado de forma apropriada é capaz de reconhecer precocemente os grupos vulneráveis e os fatores de risco relacionados à morbidade materna, tendo a capacidade de contribuir na prevenção de desfechos graves por meio de condutas clínicas e tratamentos adequados ${ }^{2,4}$.

É importante ressaltar que esse estudo apresenta algumas limitações, nomeadamente a busca bibliográfica restringida a três bases de dados, quando outras como EMBASE, EBSCO e SCOPUS também poderiam ter sido consultadas. Entretanto, o uso do descritor específico "complicações na gravidez" foi capaz de captar grande quantidade de estudos nos quais a complicação na gravidez foi claramente definida. Ainda, o processo de leitura e extração dos dados foi realizado de forma independente por mais de um pesquisador, o que contribuiu para a atenuação de prováveis vieses de informação. Apesar da restrição elencada, a relevância dos resultados obtidos deve ser destacada considerando a inexistência de outras revisões sistemáticas nacionais sobre a temática abordada. Nesse sentido, emerge, por meio dos resultados apresentados, o desafio para as políticas públicas de saúde de lidar com uma problemática que ameaça a vida nesse segmento da população. Vale ressaltar que a interpretação de tais achados deve considerar a inclusão de resultados derivados de análises não ajustadas.

Conclui-se que os transtornos mentais, as doenças infecciosas e a morbidade materna grave foram as principais complicações clínicas na gravidez abordadas por seus autores nos artigos desta revisão. Ainda, houve um considerável interesse na identificação das principais afecções ou causas de internação durante a gestação, citando-se com maiores frequências a Infecção do Trato Urinário, a anemia e as doenças hipertensivas. Estas intercorrências são de forte relevância no Brasil, ressaltando pontos instáveis dos serviços de saúde. Na conjuntura dos possíveis desfechos na saúde materno-infantil associados às complicações clínicas na gravidez, ressaltase a ocorrência de morbidade grave, internação pós-parto, óbito e indicadores de saúde infantil desfavoráveis (prematuridade e baixo peso ao nascer). Nesse contexto, os achados possibilitaram identificar que a frequência de gestantes com complicações potenciais de ameaça à vida é elevada no país, evidenciando a indispensabilidade de difusão da cobertura da atenção básica e a implantação de intervenções mais complexas. Dessa forma, a percepção da importância dessas ações, torna-se primordial para redução das complicações e desfechos materno-infantis nesse segmento da população.

\section{Colaboradores}

D Figueroa Pedraza trabalhou na concepção e planejamento, análise e interpretação dos dados; elaboração do rascunho e revisão crítica do conteúdo; e aprovação da versão final do manuscrito. ACL Lins trabalhou na análise e interpretação dos dados; elaboração do rascunho e revisão crítica do conteúdo; e aprovação da versão final do manuscrito. 


\section{Referências}

1. Moura BLA, Alencar GP, Silva ZP, Almeida MF. Internações por complicações obstétricas na gestação e desfechos maternos e perinatais, em uma coorte de gestantes no Sistema Único de Saúde no Município de São Paulo, Brasil. Cad Saude Publica 2018; 34(1):e00188016.

2. Lima Maíra RG, Coelho ASF, Salge AKM, Guimarães JV, Costa OS, Sousa TCC, Mattos DV, Sousa MAA. Alterações maternas e desfecho gravídico puerperal na ocorrência de óbito materno. Cad Saude Colet 2017; 25(3):324-331.

3. Oliveira LC, Costa AAR. Near miss materno em unidade de terapia intensiva: aspectos clínicos e epidemiológicos. Rev Bras Ter Intensiva 2015; 27(3):220227.

4. Rosendo TMSS, Roncalli AG. Prevalência e fatores associados ao Near Miss Materno: inquérito populacional em uma capital do Nordeste Brasileiro. Cien Saude Colet 2015; 20(4):1295-1304.

5. Nardello DM, Guimarães AMDN, Barreto IDC, Gurgel RQ, Ribeiro ERRO, Gois CFL. Óbitos fetais e neonatais de filhos de pacientes classificadas com near miss. Rev Bras Enferm 2017; 70(1):104-111.

6. Goudard MJF, Simões VMF, Batista RFL, Queiroz RCS, Brito MTSS, Coimbra LC, Martins MG, Barbieri MA, Nathasje IF. Inadequação do conteúdo da assistência pré-natal e fatores associados em uma coorte no nordeste brasileiro. Cien Saude Colet 2016; 21(4):1227-1238.

7. Rosendo TS, Roncalli AG, Azevedo GD. Prevalence of maternal morbidity and its association with socioeconomic factors: a population-based survey of a city in Northeastern Brazil. Rev Bras Ginecol Obstet 2017; 39(11):587-595.

8. Brasil. Ministério da Saúde (MS). Secretaria de Atenção à Saúde. Manual instrutivo das ações de alimentação e nutrição na Rede Cegonha. Brasília: MS; 2013.

9. Moher D, Liberati A, Tetzlaff J, Altman DG. Preferred Reporting Items for Systematic Reviews and Meta-Analyses: The PRISMA Statement. PLoS Med 2009; 6:e1000097.

10. Lima Marlise OP, Bonadio IC, Murata M. Sintomas depressivos na gestação e fatores associados: estudo longitudinal. Acta Paul Enferm 2017; 30(1):39-46.

11. Moraes EV, Campos RN, Avelino MM. Depressive Symptoms in Pregnancy: the influence of social, psychological and obstetric aspects. Rev Bras Ginecol Obstet 2016; 38(6):293-300.

12. Faisal-Cury A, Menezes PR, Quayle J, Matijasevich A. Unplanned pregnancy and risk of maternal depression: secondary data analysis from a prospective pregnancy cohort. Psychol Health Med 2016; 19(13):65-74.

13. Kassada DS, Waidman MAP, Miasson AI, Marcon SS. Prevalência de transtornos mentais e fatores associados em gestantes. Acta Paul Enferm 2015; 28(6):495502.

14. Almeida MS, Nunes MA, Camey S, Pinheiro AP, Schmidt MI. Transtornos mentais em uma amostra de gestantes da rede de atenção básica de saúde no Sul do Brasil. Cad Saude Publica 2012; 28(2):385-393.
15. Melo Junior EF, Cecatti JG, Pacagnella RC, Leite DFB, Vulcani DE, Makuch MY. The prevalence of perinatal depression and its associated factors in two different settings in Brazil. J Affect Disord 2012; 136(3):12041208.

16. Silva RA, Ores LC, Mondin TC, Rizzo RN, Moraes IGS, Jansen K, Pinheiro RT. Transtornos mentais comuns e auto-estima na gestação: prevalência e fatores associados. Cad Saude Publica 2010; 26(9):1832-1838.

17. Pereira PK, Slovisi GM, Lima LB, Legay LF. Complicações obstétricas, eventos estressantes, violência e depressão durante a gravidez em adolescentes atendidas em unidade básica de saúde. Rev Psiq Clín 2010; 37(5):216-222.

18. Silva RA, Jansen K, Souza LDM, Moraes IGS, Tomasi E, Silva GG, Dias MS, Pinheiro RT. Depression during pregnancy in the Brazilian public health care system. Rev Bras Psiquiatria 2010; 32(2):139-144.

19. Cunha ARC, Merchan-Hamann E. Sífilis em parturientes no Brasil: prevalência e fatores associados, 2010 a 2011. Rev Panam Salud Publica 2015; 38(6):479-486.

20. Moura AA, Mello MJG, Correia JB. Prevalence of syphilis, human immunodeficiency virus, hepatitis $B$ virus, and human T-lymphotropic virus infections and coinfections during prenatal screening in an urban Northeastern Brazilian population. Int J Infect Dis 2015; 39:10-15.

21. Domingues RMSM, Szwarcwald CL, Souza Junior PRB, Leal MC. Prenatal testing and prevalence of HIV infection during pregnancy: data from the "Birth in Brazil" study, a national hospital-based study. BMC Infect Dis 2015; 15:100.

22. Domingues RMSM, Szwarcwald CL, Souza Junior PRB, Leal MC. Prevalence of syphilis in pregnancy and prenatal syphilis testing in Brazil: Birth in Brazil study. Rev Saude Publica 2014; 48(5):766-774.

23. Miranda AE, Pinto VM, McFarland W, Page K. HIV infection among young parturient women in Brazil: prevalence and associated risk factors. AIDS Behav 2014; 18(Supl. 1):S50-S52.

24. Miranda AE, Pinto VM, Gaydos CA. Trichomonas vaginalis infection among young pregnant women in Brazil. Braz J Infect Dis 2014; 18(6):669-671.

25. Câmara JT, Silva MG, Castro AM. Prevalência de toxoplasmose em gestantes atendidas em dois centros de referência em uma cidade do Nordeste, Brasil. Rev Bras Ginecol Obstet 2015; 37(2):64-70.

26. Silva MG, Câmara JT, Vinaud MC, Castro AM. Epidemiological factors associated with seropositivity for toxoplasmosis in pregnant women from Gurupi, State of Tocantins, Brazil. Rev Soc Bras Med Trop 2014; 47(4):469-475.

27. Lopes-Mori FMR, Mitsuka-Breganó R, Bittencourt LHFB, Dias RCF, Gonçalves DD, Capobiango JD, Reiche EMV, Marimoto HK, Freire RL, Navarro IT. Gestational toxoplasmosis in Paraná State, Brazil: prevalence of IgG antibodies and associated risk factors. Braz J Infect Dis 2013; 17(4):405-409.

28. Vidal CEL, Carvalho MAB, Grimald IR, Reis MC, Baêta MCN, Garcia RB, Silva SAR. Morbidade materna grave na microrregião de Barbacena/MG. Cad Saude Colet 2016; 24(2):131-138. 
29. Cecatti JG, Souza RT, Pacagnella RC, Leal MC, Moura EC, Santos LMP. Maternal near miss among women using the public health system in the Amazon and Northeast regions of Brazil. Rev Panam Salud Publica 2015; 37(4/5):232-238.

30. Dias MAB, Domingues RMSM, Schilithz AOC, Nakamura-Pereira M, Diniz CSG, Brum IR, Martins AL, Theme Filha MM, Gama SGN, Leal MC. Incidência do near miss materno no parto e pós-parto hospitalar: dados da pesquisa Nascer no Brasil. Cad Saude Publica 2014; 30(Supl.):S169-S181.

31. Pacheco AJC, Katz L, Souza AS, Amorim MM. Factors associated with severe maternal morbidity and near miss in the São Francisco Valley, Brazil: a retrospective, cohort study. BMC Pregnancy Childbirth 2014; 14:91.

32. Oliveira Junior FC, Surita FG, Silva JLP, Cecatti JG, Parpinelli MA, Haddad SM, Costa ML, Pacagnella RC, Sousa MH, Sousa JP. Severe maternal morbidity and maternal near miss in the extremes of reproductive age: results from a national cross-sectional multicenter study. BMC Pregnancy Childbirth 2014; 14:77.

33. Magalhães MC, Bustamante-Teixeira MT. Morbidade materna extremamente grave: uso do Sistema de Informação Hospitalar. Rev Saude Publica 2012; 46(3):472-478.

34. Souza J, Cecatti J, Parpinelli M, Sousa M, Lago T, Pacagnella R, Camargo R. Maternal morbidity and near miss in the community: findings from the 2006 Brazilian demographic health survey. BJOG 2010; 117:1586-1592.

35. Falavina LP, Oliveira RR, Melo EC, Varela PLR, Mathias TAF. Hospitalização durante a gravidez segundo financiamento do parto: um estudo de base populacional. Rev Esc Enferm USP 2018; 52:e03317.

36. Varela PLR, Oliveira RR, Melo EC, Mathias TAF. Intercorrências na gravidez em puérperas brasileiras atendidas nos sistemas público e privado de saúde. Rev Latino-Am Enferm 2017; 25:e2949.

37. Cirelli JF, Surita FG, Costa ML, Parpinelli MA, Haddad SM, Cecatti JG. The Burden of Indirect Causes of Maternal Morbidity and Mortality in the Process of Obstetric Transition: A Cross-Sectional Multicenter Study. Rev Bras Ginecol Obstet 2018; 40(3):106-114.

38. Kale PL, Mello-Jorge MHP, Silva KS, Fonseca SC. Neonatal near miss and mortality: factors associated with life threatening conditions in newborns at six public maternity hospitals in Southeast Brazil. Cad Saude Publica 2017; 33(4):e00179115.

39. Saintrain SV, Oliveira JGR, Saintrain MVL, Bruno VZ, Borges JLN, Daher EF, Silva Jr GB. Fatores associados à morte materna em unidade de terapia intensiva. Rev Bras Ter Intensiva 2016; 28(4):397-404.

40. Ribeiro CF, Lopes VG, Brasil P, Silva LE, Ribeiro PH, Ugenti LC, Nogueira RMR. Dengue during pregnancy: association with low birth weight and prematurity. Rev Inst Med Trop 2016; 58:8.

41. Zanardi DM, Moura EC, Santos LP, Leal LC, Cecatti JG. The effect of maternal near miss on adverse infant nutritional outcomes. CLINICS 2016; 71(10):593599.
42. Menezes LO, Pinheiro RT, Quevedo LA, Oliveira SS, Silva RA, Pinheiro KAT, Espírito Santo GC, Jansen K. $\mathrm{O}$ impacto do baixo peso ao nascer relacionado à depressão gestacional para o financiamento federal da saúde pública: uma análise do Município de Pelotas, Rio Grande do Sul, Brasil. Cad Saude Publica 2012; 28(10):1939-1948.

43. Silva JMP, Fonseca SC, Dias MAB, Izzo AS, Teixeira GP, Belfort PP. Conceitos, prevalência e características da morbidade materna grave, near miss, no Brasil: revisão sistemática. Rev Bras Saude Mater Infant 2018; 18(1):37-65.

44. Paixão ES, Teixeira MG, Costa MCN, Rodrigues LC. Dengue during pregnancy and adverse fetal outcomes: a systematic review and meta-analysis. Lancet Infect Dis 2016; 16(7):857-865.

45. Azevedo WF, Diniz MB, Fonseca ESVB, Azevedo LMR, Evangelista CB. Complicações da gravidez na adolescência: revisão sistemática da literatura. Einstein 2015; 13(4):618-626.

46. Figueroa Pedraza D, Araujo EMN. Internações das crianças brasileiras menores de cinco anos: revisão sistemática da literatura. Epidemiol Serv Saude 2017; 26(1):169-182.

47. Truijens SEM, Spek V, van Son MJM, Guid Oei S, Pop VJM. Different patterns of depressive symptoms during pregnancy. Arch Womens Ment Health 2017; 20(4):539-546.

48. Shakeel N, Eberhard-Gran M, Sletner L, Slinning K, Martinsen EW, Holme I, Jenum AK. A prospective cohort study of depression in pregnancy, prevalence and risk factors in a multi-ethnic population. $B M C$ Pregnancy Childbirth 2015; 15:5.

49. Sion MY, Harlev A, Weintraub AY, Sergienko R, Sheiner E. Is antenatal depression associated with adverse obstetric and perinatal outcomes? J Maternal Fetal Neonatal Med 2016; 29(6):863-867.

50. Rurangirwa AA, Mogren I, Ntaganira J, Govender K, Krantz G. Intimate partner violence during pregnancy in relation to non-psychotic mental health disorders in Rwanda: a cross-sectional population-based study. BMJ Open 2018; 8:e21807.

51. Abdelrahim NA, Bayoumi MA, Ahmed HI, Fadl-Elmula IM, Homeida MM. Sexually transmitted infections other than HIV/AIDS among women of low socio-economic class attending antenatal clinics in Khartoum, Sudan. Int J STD AIDS 2016; 28(8):781787.

52. Josep Davey DL, Shull HI, Billings JD, Wang D, Adachi K, Klausner JD. Prevalence of curable sexually transmitted infections in pregnant women in Low-and Middle-Income Countries from 2010 to 2015: a Systematic Review. Sex Transm Dis 2016; 43(7):450-458.

53. Silver BJ, Guy RJ, Kaldor JM, Jamil MS, Rumbold AR. Trichomonas vaginalis as a cause of perinatal morbidity: a Systematic Review and Meta-Analysis. Sex Transm Dis 2014; 41(6):369-376.

54. Desale M, Thinkhamrop J, Lumbiganon P, Qazi S, Anderson J. Ending preventable maternal and newborn deaths due to infection. Best Pract Res Clin Obstet Gynaecol 2016; 36:116-130. 
55. Pinto VM, Basso CR, Barros CRS, Gutierrez EB. Fatores associados às infecções sexualmente transmissíveis: inquérito populacional no município de São Paulo, Brasil. Cien Saude Colet 2018; 23(7):2423-2432.

56. Padovani C, Oliveira RR, Pelloso SM. Syphilis in during pregnancy: association of maternal and perinatal characteristics in a region of southern Brazil. Rev Latino-Am Enferm 2018; 26:e3019.

57. Flatt A, Shetty N. Seroprevalence and risk factors for toxoplasmosis among antenatal women in London: a re-examination of risk in an ethnically diverse population. Eur J Public Health 2013; 23(4):648-652.

58. Laboudi M. Review of toxoplasmosis in Morocco: seroprevalence and risk factors for toxoplasma infection among pregnant women and HIV- infected patients. Pan Afr Med J 2017; 27:269.

59. Alzaheb RA. Seroprevalence of Toxoplasma gondii and its associated risk factors among women of reproductive age in Saudi Arabia: a systematic review and meta-analysis. Int J Womens Health 2018; 10:537-544.

60. Mizani A, Alipour A, Sharif M, Sarvi S, Amouei A, Shokri A, Rahimi M, Hosseini SA, Daryani A. Toxoplasmosis seroprevalence in Iranian women and risk factors of the disease: a systematic review and meta-analysis. Trop Med Health 2017; 45:7.

61. Pacagnella RC, Cecatti JG, Parpinelli MA, Sousa MH, Haddad SM, Costa ML, Souza JP, Pattinson RC. Delays in receiving obstetric care and poor maternal outcomes: results from a national multicentre cross-sectional study. BMC Pregnancy Childbirth 2014; 14:159.

62. Creanga AA, Bateman BT, KuklinaEV, Callaghan WM. Racial and ethnic disparities in severe maternal morbidity: a multistate analysis, 2008-2010. Am J Obstet Gynecol 2014; 210(5):435.e1-8.

63. Chantry AA, Deneux-Tharaux C, Bonnet M, Bouvier-Colle M. Pregnancy-Related ICU Admissions in France: Trends in Rate and Severity, 2006-2009. Crit Care Med 2015; 43(1):78-86.
64. Barry Y, Deneux-Tharaux C, Saucedo M, Goulet V, Guseva-Canu I, Regnault N, Chantry AA. Maternal admissions to intensive care units in France: Trends in rates, causes and severity from 2010 to 2014 . Anaesth Crit Care Pain Med 2019; 38(4):363-369.

65. Wanderer JP, Leffert LR, Mhyre JM, Kuklina EV, Callaghan WM, Bateman BT. Epidemiology of Obstetric-Related ICU Admissions in Maryland: 1999-2008. Crit Care Med 2013; 41(8):1844-1852.

66. Bendix J, Hegaard HK, Langhoff-Roos J, Bergholt T. Changing prevalence and the risk factors for antenatal obstetric hospitalizations in Denmark 2003-2012. Clin Epidemiol 2016; 8:165-175.

67. Bramham K, Parnell B, Nelson-Piercy C, Seed PT, Poston L, Chappell LC. Chronic hypertension and pregnancy outcomes: systematic review and meta-analysis. BMJ 2014; 348:g2301.

68. Veras TCS, Mathias TAF. Principais causas de internações hospitalares por transtornos maternos. Rev Esc Enferm USP 2014; 48(3):401-408.

Artigo apresentado em 24/08/2019

Aprovado em 21/11/2019

Versão final apresentada em 23/11/2019

Editores-chefes: Romeu Gomes, Antônio Augusto Moura da Silva 